\title{
Reasoning Exercises in Assisted Living: a cluster randomized trial to improve reasoning and everyday problem solving
}

This article was published in the following Dove Press journal:

Clinical Interventions in Aging

25 June 2014

Number of times this article has been viewed

\author{
Kristine Williams' \\ Ruth Herman ${ }^{2}$ \\ Daniel Bontempo 3 \\ 'College of Nursing, University \\ of lowa, lowa City, IA, USA; ${ }^{2}$ School \\ of Nursing, University of Kansas \\ Medical Center, Kansas City, KS, USA; \\ ${ }^{3}$ The Schiefelbusch Institute for Life \\ Span Studies, University of Kansas, \\ Dole Human Development Center, \\ Lawrence, KS, USA
}

\begin{abstract}
Purpose of the study: Assisted living (AL) residents are at risk for cognitive and functional declines that eventually reduce their ability to care for themselves, thereby triggering nursing home placement. In developing a method to slow this decline, the efficacy of Reasoning Exercises in Assisted Living (REAL), a cognitive training intervention that teaches everyday reasoning and problem-solving skills to AL residents, was tested.
\end{abstract}

Design and methods: At thirteen randomized Midwestern facilities, AL residents whose Mini Mental State Examination scores ranged from 19-29 either were trained in REAL or a vitamin education attention control program or received no treatment at all. For 3 weeks, treated groups received personal training in their respective programs.

Results: Scores on the Every Day Problems Test for Cognitively Challenged Elders (EPCCE) and on the Direct Assessment of Functional Status (DAFS) showed significant increases only for the REAL group. For EPCCE, change from baseline immediately postintervention was $+3.10(P<0.01)$, and there was significant retention at the 3 -month follow-up $(d=2.71 ; P<0.01)$. For DAFS, change from baseline immediately postintervention was $+3.52(P<0.001)$, although retention was not as strong. Neither the attention nor the no-treatment control groups had significant gains immediately postintervention or at follow-up assessments. Post hoc across-group comparison of baseline change also highlights the benefits of REAL training. For EPCCE, the magnitude of gain was significantly larger in the REAL group versus the no-treatment control group immediately postintervention $(d=3.82 ; P<0.01)$ and at the 3-month follow-up $(d=3.80$; $P<0.01)$. For DAFS, gain magnitude immediately postintervention for REAL was significantly greater compared with in the attention control group $(d=4.73 ; P<0.01)$.

Implications: REAL improves skills in everyday problem solving, which may allow AL residents to maintain self-care and extend AL residency. This benefit is particularly important given the growing population of AL residents at risk for cognitive and self-care decline.

Keywords: cognitive training, assisted living, self-care, functional decline

\section{Introduction}

Assisted living (AL) facilities are designed to meet the needs of the rapidly expanding aging population and are the fastest growing residential care option. ${ }^{1}$ One million older adults live in AL today, a number expected to double in the next decade. ${ }^{2,3}$ This growing popularity reflects the perception that AL care supports independence, autonomy, dignity, and privacy for older adults and offers a less institutional environment than a traditional nursing home $(\mathrm{NH}) .{ }^{1,4,5}$ Nevertheless, little research focuses on developing methods for optimizing and maintaining independence in frail AL residents. ${ }^{6}$

AL serves older adults who need limited assistance with instrumental activities of daily living (IADLs), such as medication administration, meal preparation, and laundry,
Correspondence: Kristine Williams 446 College of Nursing, University of lowa, 50 Newton Road, lowa City, IA 52240, USA

Email kristine-n-williams@uiowa.edu 
and lesser assistance with ADLs, such as bathing, toileting, and dressing. AL residents frequently have experienced declines in cognitive, physical, and self-care abilities and are at risk for ongoing loss and disability as a result of disease progression and institutional environments. Up to two-thirds of AL residents have early-stage Alzheimer's disease or other dementias. ${ }^{7,8}$ In contrast, NH care typically serves persons with more severe dementia. ${ }^{7}$ AL residents show significant rates of mild cognitive impairment, which only minimally impairs their daily functioning. Thus, AL staff members are primarily needed for supervision and support of the more cognitively demanding IADLs. ${ }^{9,10}$

Ninety-eight percent of AL residents prefer to remain in AL and avoid NH placement, ${ }^{11}$ but progressive declines in cognition and self-care often necessitate eventual transfer to a NH. ${ }^{4,12,13}$ Thus, AL residents fear losing their cognitive ability and becoming disabled, a fate frequently mirrored by other residents in their midst. ${ }^{13-15}$ The average length of AL residency is only $1-3$ years, ${ }^{1}$ with $25 \%-30 \%$ of residents leaving AL yearly. ${ }^{16,17}$ In addition to being a personal preference of residents, the cost of AL is less than half that of a NH. A 2012 industry sponsored survey found that yearly costs for $\mathrm{NH}$ care were $\$ 73,000$ compared with $\$ 39,600$ for AL care. ${ }^{18}$

\section{Everyday competency and cognitive interventions}

The Model of Everyday Competence ${ }^{19}$ identifies factors that elders require to solve problems and achieve self-care in seven IADLs for independent living: finances, medication, telephone use, shopping, meal preparation, housekeeping, and transportation. ${ }^{20}$ For the current study, this framework was expanded to include everyday problem-solving concerning ADLs (eg, bathing and toileting), activities with which AL residents may require assistance. This study targeted ways to improve reasoning and everyday problem-solving skills related to self-care competency because AL residents must maintain a threshold level of self-care competence or be transferred to the more costly and intensive NH care. For example, AL may require that a resident can independently transfer when faced with emergency situations.

Typically, reasoning, processing speed, and applied problem-solving decline in a person's $80 \mathrm{~s},{ }^{21}$ and these age-related cognitive changes may contribute to self-care disability. Everyday competence in IADLs and ADLS requires an array of cognitive abilities. ${ }^{22,23}$ For example, to follow a dietary restriction, an older adult must understand the restriction, remember it at meal times, and make appropriate food choices. Similarly, competent self-care requires the interplay of several critical elements: executive function, higher-order thinking and reasoning, and applied problem solving. ${ }^{24}$ Thus, the maintenance of cognitive performance, everyday competencies, and self-care skills should be targeted by interventions meant to help older adults extend semi-independent living in AL.

Training in specific cognitive skills can improve older adults' memory, processing speed, spatial orientation, reasoning, and executive function. Cognitive training, such as the intervention tested in this study, likely supports self-care, a key determinate of an older adult's ability to remain in AL. Cognitive training also benefits persons with dementia and mild cognitive decline. A meta-analysis of cognitive training research involving persons with early-stage AD reported overall effect sizes of 0.47 for interventions targeting learning, memory, executive function, ADLs, problemsolving, depression, and self-rated functioning. ${ }^{25}$ Thus, the increasing population of AL residents with cognitive decline or mild dementia would likely benefit from cognitive interventions. ${ }^{26}$

This study tested the effects of a specifically tailored cognitive-training intervention on AL residents' everyday problem-solving and self-care competence (including both ADL and IADLs). Individual and facility factors that influence problem solving and self-care (ie, physical, cognitive, and psychological health); social, family, and staff support; and AL characteristics (size, location, and affiliation) were assessed as potential covariate factors. ${ }^{27}$ Costs of providing this cognitive training intervention in $\mathrm{AL}$ were also explored.

\section{Materials and methods Study design}

A multisite, randomized, repeated-measures design compared three groups (intervention, attention control, and no-treatment control) to assess the efficacy of Reasoning Exercises in Assisted Living (REAL) in improving everyday problem solving and maintaining self-care. The design (see Table 1) combined a between-subjects factor (random assignment to treatment, attention control, or no treatment control group) with a within-subjects factor (repeated measures over time). Immediate postintervention, 3-month, and 6-month betweengroup comparisons determined the effects of REAL on problem-solving and self-care. 
Table I Research design: data collection (OI-O4) by experimental group

\begin{tabular}{llllll}
\hline Random assignment by facility & Baseline & Week 2-5 & Week 6 & Week I 2-I4 (3 month) & Week 26 (6 month) \\
\hline $\begin{array}{l}\text { Reasoning Exercises in Assisted Living }(n=25), \\
\text { experimental group }\end{array}$ & OI & $X$ & O2 & O3 & O4 \\
VITAMIN ( $n=25)$, placebo group & OI & $V$ & O2 & O3 & O4 \\
CONTROL (n=25), no-treatment control & OI & & O2 & O3 & O4 \\
\hline
\end{tabular}

Abbreviations: X, Reasoning Exercises in Assisted Living (6 individual I hour sessions); V, VITAMIN (6 individual I hour sessions); OI, Every Day Problems Test for Cognitively Challenged Elders, self-care (Direct Assessment of Functional Status and Resident Functional Capacity Screen [RFCS]), efficacy, strategy use, comorbidity, cognition, depression, support, demographics; O2, Every Day Problems Test for Cognitively Challenged Elders, self-care (Direct Assessment of Functional Status and RFCS), efficacy, strategy use, program completion, program evaluation; O3, Every Day Problems Test for Cognitively Challenged Elders, self-care (Direct Assessment of Functional Status and RFCS), efficacy, strategy use; O4, Every Day Problems Test for Cognitively Challenged Elders, self-care (Direct Assessment of Functional Status and RFCS), efficacy, strategy use, nursing home placement, assisted living residency.

\section{Settings}

Thirteen AL facilities responded to invitations to participate and, using a computer random number generator (cluster randomization), were assigned to the REAL training program (REAL), an attention control vitamin/nutritioneducation program (VITAMIN), or a no-treatment control group (CONTROL). Cluster randomization by facility was used to avoid contamination within each facility resulting from conversations among participants in different groups. The facilities represented not-for-profit and for-profit corporate chains, as well as an independent, religiously based facility. One invited facility declined to participate because of lack of interest by residents. These facilities ranged in size from 40-124 residents, were located in eastern Kansas, and included both urban and rural locations. The study was approved by the university's institutional review board and was individually approved by each AL facility.

\section{Participants}

The participants included 89 residents, ranging in age from 60-95 years, who had mild impairment in cognition, expressed concern about cognitive changes, or mild dementia. The mean Mini Mental State Examination (MMSE) score was 25.3. AL staff obtained permission to contact either residents with cognitive impairments listed in their clinical record or their surrogate decision makers.

Interested residents who expressed concerns about changes in cognition were screened before enrollment in the study. The first inclusion criterion was an 8-item Ascertain Dementia (AD8) score of 2 or more. The AD8 is an 8-item screen designed to distinguish elders with normal cognition from those with mild cognitive decline; it correlates well with the standard Clinical Dementia Rating scale, a comprehensive neuropsychological test. Internal consistency is excellent (Cronbach's alpha $=0.84) .{ }^{28}$ This screening measure queries the respondent about noticed changes in cognitively related activities such as remembering names and appointments and balancing a checkbook.

An additional inclusion criteria was a MMSE score between 19-29. ${ }^{29}$ The MMSE was administered to resident volunteers who scored 2 or higher on the AD8. Residents scoring 19-29 on the MMSE were invited to participate. Thus, participants ranged from having documented cognitive decline or mild impairments in cognition to being at risk for (and concerned about) cognitive impairment. This range was selected to ensure participants were at risk for or experiencing beginning declines in cognition but had sufficient cognitive abilities to benefit from the intervention. Residents were excluded if they did not speak and understand English or had psychiatric disorders (ie, hallucinations, schizophrenia) or physical conditions (ie, blindness) that limited their ability to participate.

Sample size was determined by a power analysis according to a pilot study in which participants gained an average of 6.6 points (standard deviation $=8.17$ ) on the Every Day Problems Test for Cognitively Challenged Elders (EPCCE) ${ }^{30}$ Power and precision calculations indicated that each group needed 25 participants; this sample size achieves $83 \%$ power to detect significant changes in the outcome measures.

\section{Procedures}

One research team member obtained consent and baseline measures. REAL and VITAMIN interventions were then administered to participants individually by trained research assistants who had graduate preparation and gerontological expertise. Before each session, the research assistants ensured the lighting was adequate, background noise was minimal, and any hearing or visual aids were in place. All interventions were provided twice weekly, over the course of 3 weeks, allowing for practice between sessions. Study 
activities took place in resident apartments to ensure privacy and minimize distractions.

\section{Interventions}

REAL

REAL provides training and practice in reasoning and problem-solving skills that promote the level of self-care needed to remain in AL residency. ${ }^{30}$ Reasoning skills were targeted on the basis of the success of the Advanced Cognitive Training in Vital Elders inductive reasoning intervention, which not only improved community-dwelling elders' reasoning performance but also reduced expected declines in IADL function over the course of 5 years. ${ }^{31}$ Inductive reasoning includes identifying patterns that can be applied to new situations; examples include identifying what comes next in a series. Reasoning strategies include scanning information, reading aloud, underlining or highlighting key or repeated information, and using lines to separate information into smaller units or chunks.

In contrast to the word and number-series problems used in Advanced Cognitive Training in Vital Elders, REAL incorporates inductive reasoning strategies (scanning, reading aloud, underlining key or repeating information, and separating information into chunks) and applies them to everyday situations related to AL residents' interests and self-care needs. For example, reasoning strategies may be applied to looking up a telephone number using several strategies: scanning the directory page for the desired name, reading aloud or underlining as a way to focus on the identified number, or breaking the seven digits into chunks to help manage the information. These strategies are introduced initially and repeated during the exercises in each subsequent session.

REAL also incorporates a framework for successful problem-solving in aging by introducing participants to problem-solving steps: identify the problem, identify the
Table 2 Reasoning Exercises in Assisted Living content outline

\begin{tabular}{ll}
$\begin{array}{l}\text { Intervention } \\
\text { session }\end{array}$ & Session title \\
\hline I & Introduction to reasoning and problem-solving skills \\
2 & Scheduling activities \\
3 & Medications \\
4 & Eating out \\
5 & Nutrition choices \\
6 & Review and challenging exercises \\
\hline
\end{tabular}

goal for problem solving (outcome), and try to solve the problem in small steps (selectively using the reasoning strategies). ${ }^{32}$ See Table 2 for the REAL content outline and Table 3 for a sample activity. These training materials were presented in a workbook format, with colorful illustrations and large (20 point) font sizes.

\section{VITAMIN}

The vitamin education attention control intervention was adapted from National Institutes of Health-funded clinical trials testing educational interventions with patients, using total parenteral nutrition. ${ }^{33}$ It mimics REAL without educating participants in reasoning strategies and problem-solving steps or involving them in skill practice, the hypothesized mechanism of REAL. In contrast, the REAL intervention introduces reasoning strategies and problem-solving steps with guided practice in applying these techniques to realworld situations. The VITAMIN intervention used colorful handouts and written materials delivered by a protocol guide. This attention control intervention provides the same amount of interventionist time as the REAL program, and subjects were assessed at equivalent intervals. Content was limited to education on the vitamin content of foods (ie, vitamins $\mathrm{C}$, B-complex, and fat-soluble intake), which was considered a neutral health topic. This content is relevant to older adults living in AL and was designed to control for potential attention, socialization, and Hawthorne effects. ${ }^{34}$

Table 3 Sample of Reasoning Exercises in Assisted Living activity

\begin{tabular}{llll}
\hline $\begin{array}{l}\text { Ultraviolet } \\
\text { index number }\end{array}$ & $\begin{array}{l}\text { Exposure } \\
\text { level }\end{array}$ & $\begin{array}{l}\text { Time to burn, } \\
\text { minutes }\end{array}$ & Actions to take \\
\hline $0,1,2$ & Minimal & 60 & Apply SPF I5 sunscreen \\
3,4 & Low & 45 & Apply SPF I5 sunscreen; wear a hat \\
5,6 & Moderate & 30 & Apply SPF I5 sunscreen; wear a hat \\
$7,8,9$ & High & $15-25$ & Apply SPF I5-30 sunscreen; wear a hat and sunglasses \\
10 or higher & Very high & 10 & Apply SPF 30 sunscreen; wear a hat, sunglasses, and protective clothing \\
\hline
\end{tabular}

The US National Weather Service issues daily forecasts of ultraviolet levels.

If the ultraviolet index is 4 , what should you do to protect yourself outdoors?

If the ultraviolet Index rises to 10, can you use the same sunscreen SPF?

What additional precautions should you take? 


\section{CONTROL}

The no-treatment group controlled for practice effects of the repeated assessments and increased power and identified maturation effects. ${ }^{35}$ This group received no interventions (or interventionist visits), but they did complete the four assessments during the 6-month study period.

REAL and VITAMIN interventions were provided to residents on an individual basis by research team members; the team included a cognitive psychologist, graduate students in gerontology and speech pathology, and masterslevel gerontological nurses with long-term-care expertise. One-on-one training and guided practice with feedback was based on a detailed training manual developed for both REAL and VITAMIN interventions. In addition, $10 \%$ of study training sessions were observed or recorded and scored using a treatment fidelity checklist. ${ }^{36-38}$ The team members were retrained if their compliance with the protocol fell below $90 \%$.

Assessment data were collected in 1-1.5 hour sessions during the week before the study began, after 4 weeks (immediately post-training), and after 3 and 6 months. A different research team member completed each assessment, so that assessors were blinded to the participant's prior performance. The research team was trained to administer assessments during an all-day class that included supervised practice and feedback. To ensure consistency, $10 \%$ of assessment sessions were observed using a checklist developed from key administration components. Assessments were collected at the same time of day for each subject (within a 2 hour window) to control for diurnal variations such as alertness. Participants originally assigned to the attention control or no-treatment control groups were invited to participate in REAL training after completing the study.

\section{Primary outcomes}

The primary outcomes were the EPCCE and Direct Assessment of Functional Status (DAFS). It was hypothesized that EPCCE and DAFS scores would be higher in the REAL group compared with in the control groups postintervention and after 3 and 6 months.

EPCCE is a 32-item measure that tests global cognitive processes and higher-order executive functions in the performance of IADLs. ${ }^{39,40}$ EPCCE presents 16 written stimuli (eg, a prescription label), each of which is followed by two problem-solving questions. Difficulty increases through the test. Domains related to the complex IADL tasks tested include finances, medications, transportation, telephone use, household management, and meal preparation. Although the EPCCE focuses on solving problems specific to IADL self-care, similar abilities are needed to accomplish ADLs. Because the IADLs generally require more complex cognitive skills, they provide a sensitive measure for self-care in general.

EPCCE reliability is reported at 0.90 (Cronbach's alpha). Scores on EPCCE also correlate with the ability to perform IADL tasks, as well as caregiver reports. ${ }^{39}$ EPCCE is used extensively in research involving frail and cognitively impaired older adults. ${ }^{40-42}$ If the EPCCE is repeatedly administered, then a practice effect may arise; however, the long intervals between the assessments make this effect unlikely.

The DAFS measures self-care competencies and is a direct-observation measure designed to overcome any overestimation of functional abilities and inaccurate recall. ${ }^{43}$ It assesses behaviors during simulated $\mathrm{ADL} / \mathrm{IADL}$ tasks. The DAFS has been validated across elder care settings, including $\mathrm{NH}$ and dementia care populations, where it effectively discriminated normal elders from those with dementia and depression. ${ }^{44}$ It assesses time orientation, communication, transportation, finances, shopping, grooming, and eating. Within each area, specific tasks simulate real-world activities. For instance, the subject is asked to tell time at four progressively difficult clock settings. Scores range from $0-103$, with higher scores indicating higher functioning. Intraclass correlations of 0.85 and interrater reliability of 0.099 are reported. ${ }^{45,46}$

\section{Baseline descriptive data}

Age, race and ethnicity, marital status, hospitalizations, diagnoses, medications, hearing, communication, and cognitive impairment data were collected from the AL medical record to describe participants and potentially control for group differences. MMSE and Modified Mini Mental Status $(3 \mathrm{MS})^{47}$ scores were collected to assess cognitive status (see Table 4).

Additional factors with the potential to influence intervention effectiveness were also measured and are included in Table 4. The Modified Cumulative Illness Rating Scale was completed as a measure of comorbidities. ${ }^{48}$ The Patient Health Questionnaire was selected to measure depression because of its nonthreatening presentation and its fit with problem-solving and self-care. ${ }^{49}$ Educational level was also assessed through review of AL records, although approximately $42 \%$ of records did not provide this information.

Four facilities each participated in REAL and the notreatment control groups, and five were included in the 
Table 4 Baseline characteristics and assessment scores of assisted living residents

\begin{tabular}{|c|c|c|c|}
\hline Characteristic & $\begin{array}{l}\text { Reasoning Exercises in } \\
\text { Assisted Living group }\end{array}$ & $\begin{array}{l}\text { VITAMIN } \\
\text { group }\end{array}$ & $\begin{array}{l}\text { CONTROL } \\
\text { group }\end{array}$ \\
\hline Age, years, mean (standard deviation) & $86(5.9)$ & $83(10.5)$ & $86(4.8)$ \\
\hline \multicolumn{4}{|l|}{ Sex } \\
\hline Male & $12(4 \mid \%)$ & $6(21 \%)$ & II (34\%) \\
\hline Female & $17(59 \%)$ & $22(79 \%)$ & $21(66 \%)$ \\
\hline \multicolumn{4}{|l|}{ Race/ethnicity } \\
\hline Black & I (3\%) & $\mathrm{I}(4 \%)$ & 0 \\
\hline White & $28(97 \%)$ & $27(96 \%)$ & $32(100 \%)$ \\
\hline Married & $5(17 \%)$ & $4(14 \%)$ & $12(38 \%)$ \\
\hline Education, years, mean (standard deviation) & $13.30(2.6)$ & $15.65(1.8)$ & $13.56(2.4)$ \\
\hline Mini Mental State Examination score, mean (standard deviation) & $25.6(2.7)$ & $25.7(2.7)$ & $24.7(3.1)$ \\
\hline Modified Mini Mental Status score, mean (standard deviation) & $85.14(9.5)$ & $84.29(9.9)$ & $83.91(10.4)$ \\
\hline $\begin{array}{l}\text { Every Day Problems Test for Cognitively Challenged Elders score, } \\
\text { mean (standard deviation) }\end{array}$ & $12.69(7.6)$ & $18.00(8.3)$ & $13.34(8.1)$ \\
\hline Direct Assessment of Functional Status score, mean (standard deviation) & $93.4 \mathrm{I}(7.7)$ & $94.14(6.4)$ & $93.94(6.5)$ \\
\hline Modified Cumulative Illness Rating Scale score, mean (standard deviation) & $25.7(4.1)$ & $24.4(4.0)$ & $25.8(4.2)$ \\
\hline Patient Health Questionnaire score, mean (standard deviation) & $2.76(3.5)$ & $4.07(4.7)$ & $3.03(3.4)$ \\
\hline
\end{tabular}

vitamin attention control group. One additional facility was required for the attention control group to achieve adequate sample size.

\section{Statistical methods}

Data analysis initially explored distributions and mean structures for both EPCCE and DAFS outcomes. This process used descriptive statistics and figures and then subsequently fitted a factorial group $\times$ time model for each outcome. Any significant baseline differences in Table 4 occurred despite randomization; however, subsequent models were designed to control for baseline differences.

\section{Primary analyses}

The main analysis entailed multilevel modeling of EPCCE and DAFS assessments by treatment group (REAL, VITAMIN, CONTROL) and time (baseline; "post," at 1 month; and follow-ups at 3 and 6 months; ie, month $=0,1,4,7$ ). Multilevel modeling (also called mixed-effects modeling, hierarchical linear modeling, or random coefficient modeling) is an advantageous method when handling dependencies in repeated measures data because incomplete data (ie, dropout) are easily accommodated. ${ }^{50-53}$ Moreover, for analysis of group randomized trials with more than two time points, multilevel modeling is the recommended approach. ${ }^{54}$

A series of models examined fixed effects for initial group differences, covariate baseline controls (cognitive status, age, depression, morbidity), changes over time (response to training and subsequent retention), random effects (individual differences in response), and the effect of dropout. Because the study excluded participants who did not complete the training or completed only the baseline session, dropout is only evidenced in the 3- and 6-month follow-up assessments. EPCCE and DAFS models computed using only data from participants who completed all assessments did not significantly differ from the models including data from all participants, regardless of dropout.

Initial models contained both a random intercept for facility (level 3) and a random intercept for subjects (level 2) nested in facilities. After entering fixed effects into DAFS models, the facility (level 3) variance estimate was reduced to virtually zero, and subsequent analysis continued with two-level (occasion nested within subjects) models.

After entering baseline controls, the facility-level variance estimate in DAFS modeling fell to $2.75 \mathrm{e}^{-18}$, and a standard error failed to estimate. Corresponding facility intraclass correlation coefficient (ICC) was 9.17e-20, with 0 standard error. Retaining the three-level framework despite zero variance at the third level, some models failed to converge (especially testing random level-two slopes in the final model). This is common when estimated parameters are on the boundary of the parameter space (ie, zero variance). There were no practical consequences of not modeling the zero variance at the facility level. We obtained subject-level variance estimates and ICC identical to those estimated in the three-level model. In addition, nearly identical fixed-effect estimates were obtained.

Beginning with an empty model to establish the extent of between-subject and within-subject variance, successive models introduced the main effects of group, controls for baseline values of important covariates, and finally the interaction effects of treatment groups by time. Initially, 
time was coded discretely as occasions (fully saturated), and subsequently, a more parsimonious continuous-time coding was used to yield slopes for gain at postintervention and subsequent linear change over follow-up.

The continuous model was parameterized as two linear splines: gain and loss. Spline regression is a nonparametric approach that approximates nonlinear responses across a continuous predictor without parametric assumptions/ artifacts. ${ }^{55}$ With linear splines, the effect of the explanatory variable is assumed to be piecewise linear on some number of segments demarcated by knots. ${ }^{56,57}$ Two spline segments were knotted immediately postintervention (ie, month $=1$ ), which rendered a model in which the response to treatment and the response over follow-up were both linear. Estimates for linear gain were coded for 1 month, whereas estimates for linear loss were coded to reflect net 6 months. Linear spline coefficients are therefore straightforward to interpret as immediate growth and subsequent 6-month decline.

For the continuous-time model, an ancillary model with additional random coefficients was estimated to examine individual differences in response over time (ie, random gain and loss slopes). This model was estimated twice: once using all available data, and again using only complete data (ie, present at all four measurement occasions) to gauge any dropout issues. Model evaluation was guided by loglikelihood, Akaike information criterion, Bayesian information criterion, and pseudo $R^{2}$ indices. No single index was given ultimate authority; rather, they were taken as guides along with parsimony, interpretation, and theoretical considerations.

\section{Covariate analyses}

To explore the possible effects of covariates on groupspecific gain/loss, models with interactions of covariates and group-specific gain/loss parameters were estimated. These models move beyond controlling for baseline covariate values and permit examination of subject-level factors that may moderate change over time. These interaction results and associated plots are presented with caution about power, but identification of which residents might benefit most is an important consideration.

\section{Results Descriptive}

The baseline demographics are summarized in Table 4. At the outset, the CONTROL group had more participants, but by follow-up, it also had lost more participants than either the REAL or VITAMIN groups (see Figure 1).
Despite randomization, the group means for the EPCCE were not equivalent at baseline; however, these differences can be controlled for in analysis models examining change over time. (See group mean baseline scores at the bottom of Table 4.) For the EPCCE, the baseline means of the REAL, VITAMIN, and CONTROL groups were 12.7, 18.0, and 13.3, respectively. In comparison, at baseline, the mean scores on the DAFS were approximately 94 across the groups. Despite these baseline values, immediately after training, scores in the REAL group increased, as anticipated, for both EPCCE (+3.10) and DAFS (+3.52). Change scores for EPCCE and DAFS at each occasion are shown in Table 5.

Large individual differences are evident in the standard deviations and ranges in test scores. In each group at each time, the range for both EPCCE and DAFS was approximately 25, and standard deviations were between 6 and 9; therefore, the changes to be modeled are distributional (ie, a movement of the means within the range).

\section{Multilevel models}

After controlling for covariate baseline values, the facility variance was greatly reduced in models of EPCCE and fell to zero in models of DAFS. For this reason, EPCCE models of change over time retained the random intercept for facility (ie, remained three levels), whereas the models of change over time for DAFS were estimated without a random intercept for facility (ie, were two-level models).

Table 6 summarizes the fully saturated and linear-spline models for both EPCCE and DAFS outcomes. (A full tabulation of all models is available from $\mathrm{KW}$ on request.) Covariate baseline controls for age, cognitive status, staff-rated functional status, depression, and comorbidities were found to be significant in EPCE or DAFS models. These effects were included in the models shown in Table 6 but are not tabulated. Effects were in expected directions. Additional controls for ADL/IADL function, facility size, and urban/ rural facility location did not significantly enter as controls in EPCCE or DAFS models and were not retained in any subsequent models.

The linear-spline models showed the best fit to the data. Likelihood ratio tests determined that the saturated discrete-occasion model (permitting nonlinear change over follow-up) was not significantly better than the more parsimonious linear spline model for either EPCCE (likelihood-ratio $X^{2}(3)=2.61 ; P=0.46$ ) or DAFS (likelihood-ratio $\left.X^{2}(3)=0.02 ; P=0.99\right)$. Also, there were no real differences in explained variance. At the 3-month follow-up, 


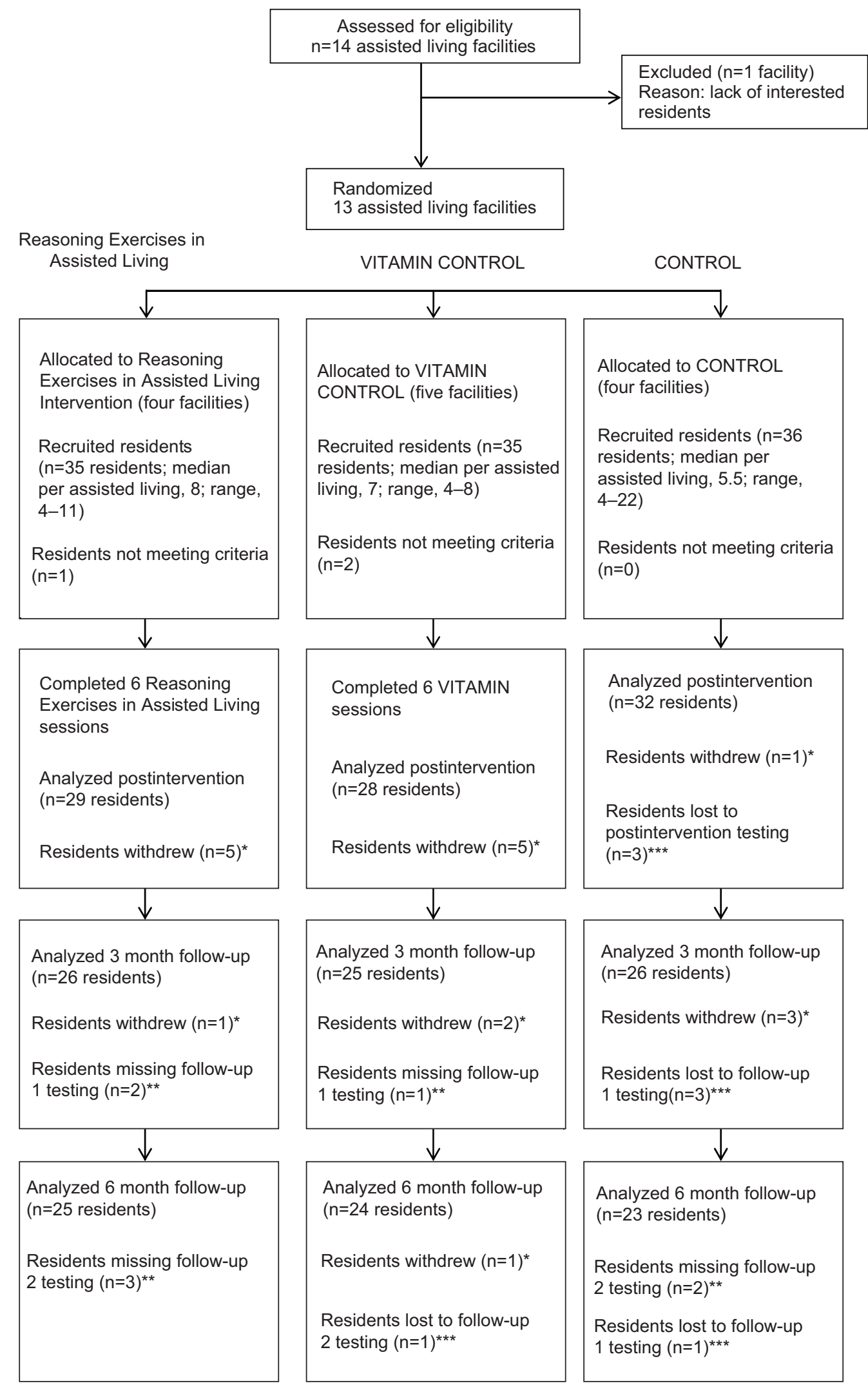

Figure I Participant flow-through study.

Notes: *Residents who withdrew voluntarily left the study and chose no longer to continue (ie, too busy, test anxiety). **Residents missing testing times were still enrolled in the study but missed testing for various reasons (ie, scheduling conflicts, hospitalization). ***Residents lost to study were no longer enrolled because of death, illness, or left facility. 
Table 5 Raw change from baseline Every Day Problems Test for Cognitively Challenged Elders and Direct Assessment of Functional Status at postintervention, 3 months, and 6 months

\begin{tabular}{|c|c|c|c|}
\hline Group/outcome & $\begin{array}{l}\text { Mean (standard } \\
\text { deviation) change } \\
\text { postintervention* }\end{array}$ & $\begin{array}{l}\text { Mean (standard } \\
\text { deviation) change } \\
\text { at } 3 \text { month follow-up* }\end{array}$ & $\begin{array}{l}\text { Mean (standard } \\
\text { deviation) change } \\
\text { at } 6 \text { month follow-up* }\end{array}$ \\
\hline \multicolumn{4}{|l|}{ Reasoning Exercises in Assisted Living } \\
\hline Every Day Problems Test for Cognitively Challenged Elders & $3.10(5.2)$ & $2.7 \mathrm{I}(5.4)$ & $1.67(5.6)$ \\
\hline Direct Assessment of Functional Status & $3.52(7.2)$ & $1.25(6.2)$ & $-0.96(4.5)$ \\
\hline \multicolumn{4}{|l|}{ VITAMIN } \\
\hline Every Day Problems Test for Cognitively Challenged Elders & $\mathrm{I} .54(5.3)$ & $0.17(4.0)$ & $0.21(4.6)$ \\
\hline Direct Assessment of Functional Status & $-1.21(5.9)$ & $-0.68(8.0)$ & $-0.43(5.9)$ \\
\hline \multicolumn{4}{|l|}{ CONTROL } \\
\hline Every Day Problems Test for Cognitively Challenged Elders & $-0.72(6.8)$ & $-1.15(6.3)$ & $0.92(5.5)$ \\
\hline Direct Assessment of Functional Status & $0.88(4.9)$ & $-0.29(3.5)$ & $-1.3 \mid(5.4)$ \\
\hline
\end{tabular}

Note: *Within-individual change from baseline.

any departures from a simple linear decline were unremarkable, although this may be because of limited power. We tabulate both models in Table 6 for ease of discussing EPCCE and DAFS estimates at 3 months.
Additional planned models focused on individual differences in change and sensitivity to dropout were not remarkable and are not tabulated. The random coefficient models estimating random gain/loss slopes showed that individual

Table 6 Saturated group by time and final spline model results

\begin{tabular}{|c|c|c|c|c|}
\hline \multirow[t]{2}{*}{ Model } & \multicolumn{2}{|c|}{$\begin{array}{l}\text { Every Day Problems Test for } \\
\text { Cognitively Challenged Elders }\end{array}$} & \multicolumn{2}{|c|}{$\begin{array}{l}\text { Direct Assessment } \\
\text { of Functional Status }\end{array}$} \\
\hline & Saturated & Spline & Saturated & Spline \\
\hline \multicolumn{5}{|l|}{ Between-group difference score } \\
\hline Control baseline (constant) & 15.375 & 15.356 & 93.912 & 93.912 \\
\hline Reasoning Exercises in Assisted Living (difference score) & -2.509 & -2.435 & 0.338 & 0.338 \\
\hline VITAMIN (difference score) & 2.415 & 2.435 & -0.948 & -0.949 \\
\hline \multicolumn{5}{|l|}{ Within-group difference score } \\
\hline CONTROL, postintervention & -0.719 & & 0.875 & \\
\hline CONTROL, 3 month & $-1.07 \mid$ & & -0.371 & \\
\hline CONTROL, 6 month & 0.962 & & -1.503 & \\
\hline Reasoning Exercises in Assisted Living, postintervention & $3.103 * *$ & & $3.517^{* * *}$ & \\
\hline Reasoning Exercises in Assisted Living, 3 months & $2.728^{* *}$ & & 1.259 & \\
\hline Reasoning Exercises in Assisted Living, 6 months & 1.642 & & -0.980 & \\
\hline VITAMIN, postintervention & 1.536 & & -1.214 & \\
\hline VITAMIN, 3 months & 0.120 & & -0.730 & \\
\hline VITAMIN, 6 months & 0.153 & & -0.476 & \\
\hline CONTROL, postintervention gain & & -1.038 & & 0.860 \\
\hline Reasoning Exercises in Assisted Living, postintervention gain & & $3.208 * * *$ & & $3.514 * * *$ \\
\hline VITAMIN, postintervention gain & & 1.321 & & -1.180 \\
\hline CONTROL, 6 month loss & & 1.573 & & $-2.384 *$ \\
\hline Reasoning Exercises in Assisted Living, 6 month loss & & $-1.44 \mid$ & & $-4.498 * * *$ \\
\hline VITAMIN, 6 month loss & & -1.415 & & 0.744 \\
\hline$R$-square & $46.4 \%$ & $46.4 \%$ & $48.3 \%$ & $48.3 \%$ \\
\hline Log likelihood & -980.623 & -981.827 & -978.215 & -978.224 \\
\hline Akaike information criterion & 1999.246 & 1995.855 & |994.43 | & 1988.448 \\
\hline Bayesian information criterion & 2071.256 & 2056.494 & 2066.440 & 2049.087 \\
\hline Variance facility & 4.658 & 4.659 & & \\
\hline Variance subject & 22.573 & 22.443 & 12.749 & $|2.75|$ \\
\hline Within-subject residual & 13.434 & 13.602 & $|6.06|$ & $|6.06|$ \\
\hline ICC facility & 0.115 & 0.113 & & \\
\hline ICC subjects & 0.670 & 0.665 & 0.443 & 0.443 \\
\hline
\end{tabular}

Notes: $* P<0.05 ; * * P<0.01$; $* * * P<0.001$.

Abbreviation: ICC, intraclass correlation coefficient. 
differences (variance) in gain or loss were similar in magnitude to between-subject differences, and there were moderate to large correlations among all three random effects. Bayesian information criterion values favored the simpler random intercept models shown in Table 6. In a model requiring data at all four occasions to check sensitivity to dropout, result patterns did not change when participants who dropped out were listwise excluded.

The models in Table 6 show that immediately after training, REAL had a significant positive effect on both EPCCE and DAFS, which weakened over time. Figure 2 presents a graphic overview of the estimates in Table 6 . The dashed lines with solid markers show the saturated groupby-occasion (discrete time) model, whereas bold lines show the results of the linear spline model. Both EPCCE and DAFS significantly increased immediately postintervention, but the significant retention evident in the EPCCE scores at 3-month follow-up was not significant in DAFS at 3 months postintervention, and although linear loss was not significant in the REAL group in EPCCE spline model, linear loss was significant in the DAFS spline model. Nevertheless, the DAFS model coefficients still reflected the expected direction and pattern. Gains declined over follow-up for both EPCCE and DAFS, but retention was better for EPCCE.
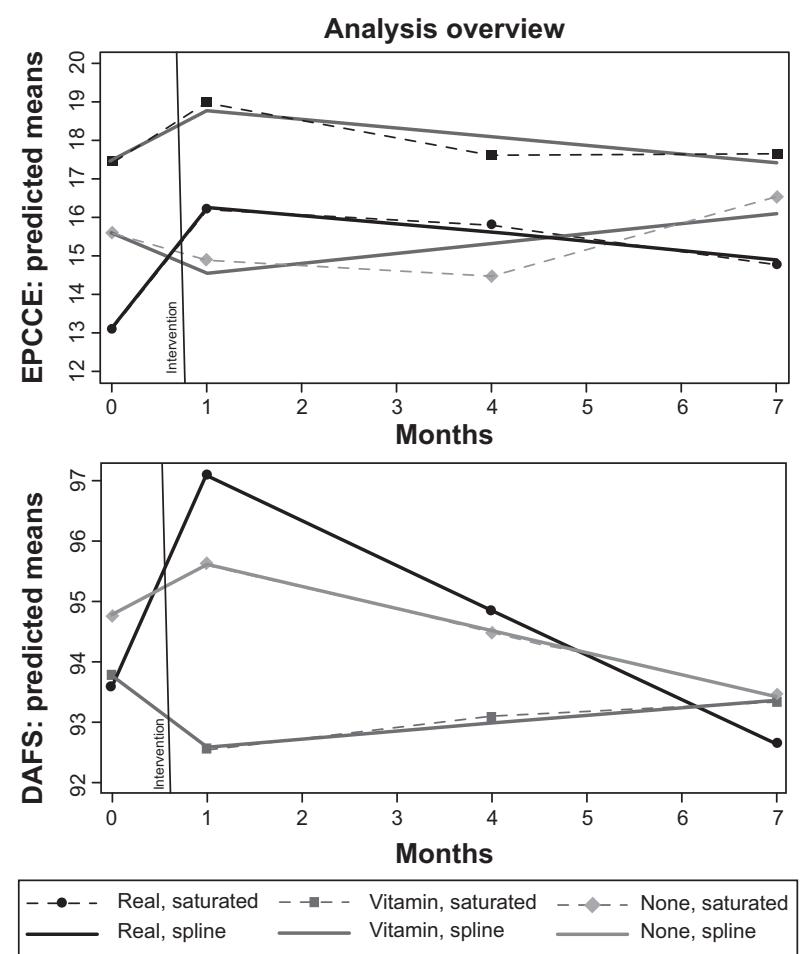

Figure 2 Linear spline models for Every Day Problems Test for Cognitively Challenged Elders (EPCCE; top) and Direct Assessment of Functional Status (DAFS; bottom) for Reasoning Exercises in Assisted Living, VITAMIN, and CONTROL groups.

\section{EPCCE}

Between-group (baseline) and group $\times$ time effects (change) in both the saturated group $\times$ time and linear spline models are evidence that initial group differences are not significant but that there were significant group-by-time effects in the REAL group. Under the model parameterization used, the group $\times$ time effect expresses how each group's time-specific mean contrasts with its own baseline (ie, within-group change). The REAL group gained a significant 3.10 points $(P<0.01)$ at postintervention and is well above the baseline at the 3-month follow-up $(d=2.73 ; P<0.01)$; at the 6-month follow-up, there is a nonsignificant gain over baseline ( $d=1.64$; nonsignificant). Because parameterization is for within-group change, the estimate at the 6-month follow-up is the group's net gain/loss over the study period, so there was a net positive but nonsignificant change for the REAL group. The linear spline model for EPCCE closely agreed in gain $(3.21 ; P<0.001)$ and 6-month loss $(-1.44$; nonsignificant).

In contrast, the VITAMIN and CONTROL groups did not show significant gains immediately postintervention. Consistent with "no treatment", the CONTROL group manifested no significant departure from its baseline at any point. In both the saturated and spline models, the VITAMIN group evidences a nonsignificant gain immediately postintervention about one-third the magnitude of the REAL group. For visualization, contrast the REAL and VITAMIN slopes between months 0 and 1 in the top panel of Figure 2. In both models, the control group is nonsignificantly lower immediately postintervention.

Importantly, the magnitude of gains and loss coefficients in the VITAMIN and CONTROL groups balance out (CONTROL, -1.04 change at postintervention and +1.57 change at 6 months, for a net -0.53 ; VITAMIN, +1.32 at postintervention and -1.42 at 6 months, for a net -0.10 ). In contrast, the gain made by the REAL group was not only significant but was more than twice the magnitude of subsequent loss ( +3.21 at postintervention and -1.44 at 6 months, for a net 1.77), although net gain was not significant.

The benefit of REAL training is also seen in post hoc across-group comparisons of EPCCE change from baseline. The magnitude of mean EPCCE gain was significantly larger in the REAL group versus the no-treatment control group immediately postintervention $(d=3.82 ; P<0.01)$ and at the 3-month follow-up $(d=3.80 ; P<0.01)$. Although the VITAMIN group mean did not significantly increase from baseline, the group's mean gain did trend larger than the no-treatment control group's mean gain $(d=2.24$; 
$P<0.1)$ immediately postintervention. The REAL group's mean gain was not significantly larger than the VITAMIN group's gain immediately postintervention $(d=1.57$; nonsignificant) but trended larger at the 3-month follow-up $(d=2.61 ; P<0.1)$.

Several developments across EPCCE models are worth noting. There were 327 observations nested in 89 participants nested in 13 facilities. Unconditional between-facility variance estimate was 15.02 (ICC $=0.20$ ), and betweensubject was 46.21 (ICC $=0.81$ ), with 14.58 residual (withinsubject) variance. After group and baseline covariates were entered, between-facility variance fell to 4.59 (ICC $=0.11$ ) and between-subject variance fell to 22.24 (ICC $=0.65)$. Cognitive status was largely responsible for decreasing facility-level variance. Pseudo $R^{2}$ was $6.9 \%$ after group was entered and rose to $45.5 \%$ after entry of baseline covariates. With the additional entry of within-person time parameters, residual variance fell to 13.43 (saturated) and 13.60 (spline); pseudo $R^{2}$ rose to $46.4 \%$ for both saturated and spline models.

\section{DAFS}

Between-group and group $\times$ time effects for DAFS are similar to EPCCE in that initial group differences are not significant and the only significant within-group baseline change occurred immediately postintervention in the REAL group (saturated, $d=3.52[P<0.001]$; spline, $d=3.51[P<0.001]$ ). This is seen in the comparatively larger rise between baseline and postintervention for the REAL group in the lower panel of Figure 2. In contrast to EPCCE, at the 3-month follow-up, the REAL group's score was higher than baseline, but the difference was not significant. REAL effects on DAFS scores were relatively smaller (than on EPCCE) and dissipated sooner. For all three groups at the 6-month follow-up, the net change was negligible.

Post hoc across-group comparisons of DAFS change from baseline are not as consistent as for EPCCE, but there is support for the benefits of REAL training. The mean change postintervention in the REAL group trended larger than for the no-treatment control group $(d=2.64 ; P<0.1)$. In addition, the comparison of the postintervention mean DAFS change in REAL versus VITAMIN groups was significant $(d=4.73 ; P<0.01)$.

Several developments across DAFS models are noted in terms of model fit and estimated variance coefficients. As with EPCCE, for DAFS there were 327 observations nested in 89 participants nested in 13 facilities. Unconditional between-facility variance estimate was 3.61 , and between-subject was 34.75, with an estimate of 17.68 for residual (within-subject) variance. After group and baseline covariates were entered, between-facility variance was virtually zero, and subsequent models were estimated as two-level (versus three-level) models. Between-subject variance fell to 12.30 . Pseudo $R^{2}$ was $0.01 \%$ after group was entered but rose to $45.9 \%$ after entry of baseline covariates. With the additional entry of within-person time parameters, residual variance fell to 16.06 in both saturated and spline models; the pseudo $R^{2}$ rose to $48.3 \%$ for both saturated and spline models.

\section{Covariate analyses}

Investigation of the interaction effects of covariates (age, cognitive status, depression, comorbidity level, and ratings of functional limitations) on REAL training benefits for everyday problem solving (EPCCE) and functional status (DAFS) was conducted by entering two-way covariate $\times$ time and three-way covariate $\times$ time $\times$ treatment terms into a series of models. (These data are not tabulated but are available from KW.) Because the only significant gain in earlier models was found in the REAL group, these analyses collapsed the VITAMIN and no-treatment control groups into a single control group (OTHER) to provide more power as additional covariates and interaction terms are modeled. Models controlled for group baseline differences (REAL versus OTHER) and were parameterized to show within-group gain/loss changes from each group's baseline. In addition, change from baseline cognitive status (3MS score) was included as a time-varying covariate (in addition to baseline cognitive status). Following earlier analyses, EPCCE models were three-level and DAFS models were two-level. As each two-way (baseline) covariate $\times$ time interaction was entered, the three-way interaction was subsequently entered only when the two-way interaction had been significant.

Some two-way covariate $\times$ gain interaction effects were found for EPCCE, but not for DAFS. No significant three-way covariate $\times$ time $\times$ REAL effects were found. For both EPCCE and DAFS, the time-varying covariate, change from baseline cognitive status, did significantly contribute in addition to baseline cognitive status and was retained in all covariate interaction models. For EPCCE, two-way covariate $\times$ gain interactions were found for both baseline cognitive status (estimate $=0.145 ; P<0.05$ ) and staff-rated functional status (estimate $=0.131 ; P<0.10$ ). No significant covariate $\times$ loss interactions were found. These interactions provide weak evidence that REAL training benefit for EPCCE 


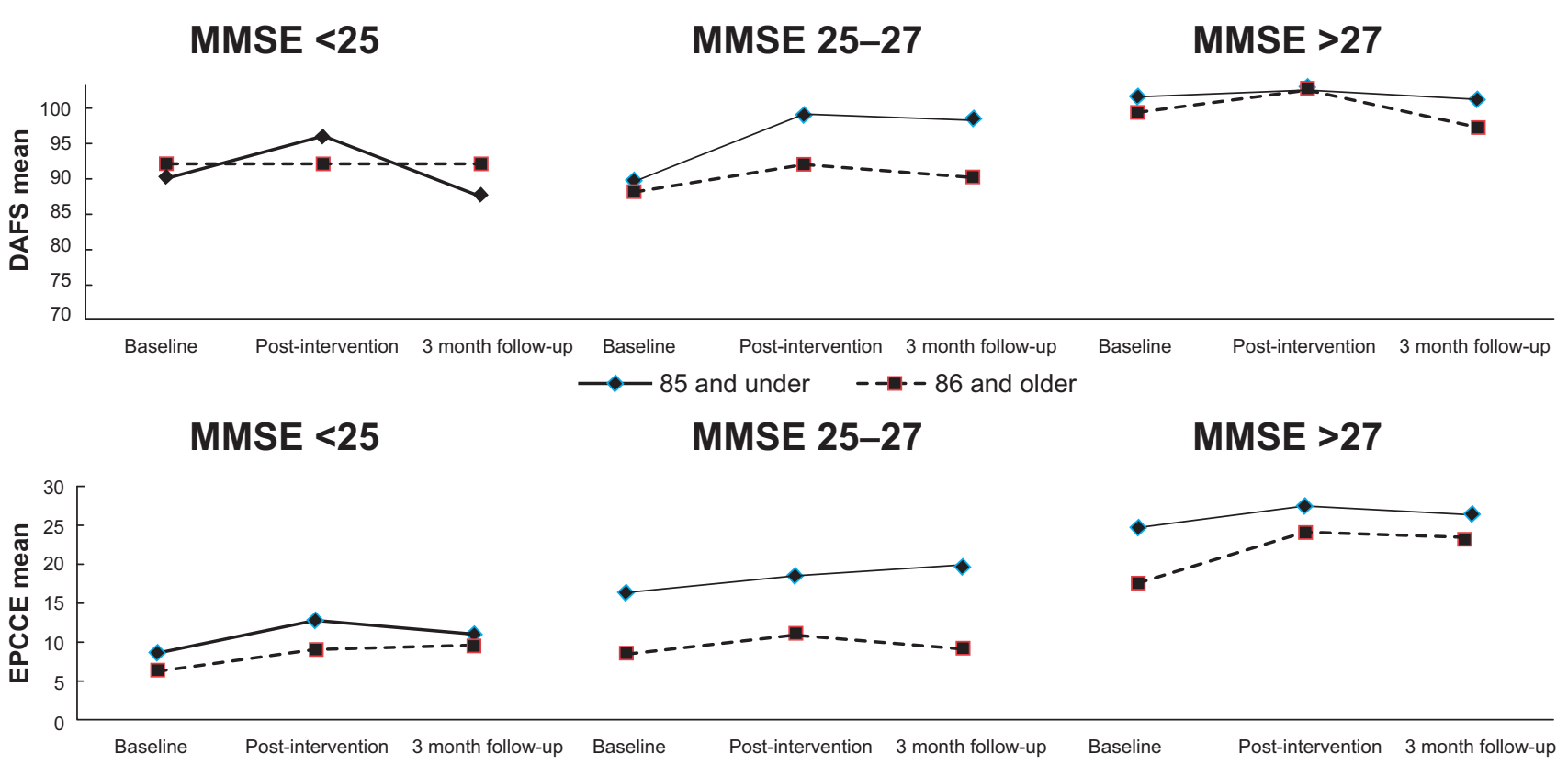

Figure 3 Raw data plots for Every Day Problems Test for Cognitively Challenged Elders (EPCCE; bottom) and Direct Assessment of Functional Status (DAFS, top) for Reasoning Exercises in Assisted Living by age and cognitive status through 3 month follow-up.

Abbreviation: MMSE, Mini Mental State Examination.

increased slightly for participants with higher baseline cognitive status and fewer staff-rated functional limitations.

Because of low power for interaction models, potential interaction of cognitive status and age was further explored in summary plots of raw EPCCE and DAFS data. Figure 3 suggests that younger elders ( $<85$ years) maintained gains in problem-solving longer. The oldest adults ( $>85$ years) with higher baseline cognition showed the highest overall gain in problem solving but may also require a booster sooner. There was a ceiling effect for DAFS in the group with the highest cognitive function (baseline MMSE > 26). EPCCE gains significantly effected DAFS gains, supporting the conceptual hypothesis that problem-solving contributes to functional performance.

\section{Program evaluation}

Overall, resident response to and rating of REAL were positive, which is a necessary feature for acceptance across AL settings. Of the participants who participated in the REAL program $(\mathrm{N}=29), 76 \%$ reported they would recommend REAL to other AL residents, whereas 24\% were unsure. When asked how often the participating residents used what they learned in the REAL program, with 1 being not used and 5 being used daily, the average mean use was 3.7, indicating that most residents in the REAL group reported applying the training regularly to their daily life. Dropout was also low in the REAL group and in comparison to the no-treatment control group, the participants in which may have tired of periodic assessments without receiving any intervention or establishing a relationship with the interventionists.

\section{Discussion}

REAL training led to gains in everyday problem solving and functional performance for AL residents, regardless of age and cognitive status. There was also evidence that increasing everyday problem solving improves self-care performance. Benefits of REAL training are evidenced in accounts of the within-group EPCCE or DAFS change from baseline, where only the REAL group showed significant gains. Benefits are also supported by across-group comparisons of change magnitude, especially for EPCCE.

\section{Primary findings}

EPCCE scores for the REAL group participants increased significantly immediately postintervention and at the 3-month follow-up assessments; this trend was maintained at the 6-month follow-up visit. Neither the VITAMIN nor the notreatment CONTROL groups showed a similar improvement. DAFS scores also improved significantly from baseline in the REAL group only. However, this effect disappeared by the 3- and 6-month reassessments. In contrast to EPCCE, each of the three groups' changes from baseline DAFS baseline scores were far less (Figure 2); nevertheless, they were controlled for using the same model parameterization described for EPCCE. 
Overall, the scores in each group had large and overlapping ranges, and in the period after the intervention, participants showed variable patterns of change in their scores. DAFS scores may have some ceiling effect because they were primarily at the top of the range. Future research may use an expanded version of DAFS, adapted for community-dwelling elders, to overcome this concern.

These results support the conceptual framework for the study. Theoretically, cognitive status directly affects everyday problem solving, which in turn affects functional status or everyday competencies. REAL effects on EPCCE (problem solving) were evident immediately and after 3 months; by the 6-month follow-up, an improvement was still detectable, but the effects had mostly dissipated. REAL effects on DAFS were evidenced only postintervention. These results suggest that a booster for the REAL intervention may be appropriate at around 3 months if the goal is to maintain functional status. This will be tested in ongoing research. Evaluation of effects on $\mathrm{NH}$ placement will require extending the time of the study and increasing the sample size (in the 6-month study, only two participants were placed).

\section{Clinical significance}

The clinical significance of REAL's effects merits careful consideration. It is important to consider the value of increased everyday problem solving (EPCCE) in terms of how this affects the life of an AL resident. Effects on selfconfidence and self-esteem were not addressed in this study but could significantly affect AL resident quality of life. The less-dramatic effects on the observed functional status measure may be more meaningful, considering that loss of self-care or increasing dependence requires more attention from staff and may prompt $\mathrm{NH}$ placement to receive a higher level of care. Indeed, many AL facilities now charge residents according to the level of care they require.

Immediate changes in functional performance may be less critical to consider compared with long-term effects on maintaining self-care that may support "aging in place" for AL residents. Ongoing research should examine the long-term effects of REAL and other cognitive interventions and establish when booster intervention sessions are needed. Ultimately, larger-scale studies are needed to assess whether REAL and other interventions might delay $\mathrm{NH}$ placement (and estimate subsequent savings from reduced costs for $\mathrm{NH}$ care).

\section{Feasibility}

Effects of the covariates yielded anticipated relationships and also suggested that REAL provides benefits to AL residents of differing ages and cognitive status. Although higher MMSE and 3MS scores at baseline were associated with higher baseline scores on both EPCCE and DAFS, only small effects on training gains were noted (and only for EPCCE), suggesting REAL benefits residents who meet the inclusion criteria, which is a large proportion of $\mathrm{AL}$ populations. Higher baseline scores for depression were associated with lower scores on both assessments; however, those participants with higher depression scores still demonstrated gains from the REAL intervention. Widespread benefits and acceptance by residents are two factors suggesting this intervention has a potential for widespread implementation.

The findings of this study support other research that finds value in cognitive training for older adults, including those experiencing both normal and abnormal age-related changes in cognition with aging. ${ }^{25,58,59}$ Our population included older adults with measurable mild impairments in cognition and early-stage dementia, as well as "worried well" older adults. Cognitive training is an important intervention for many older adults, in that improvements have been realized across cognitive levels, as reflected in populations residing in AL. Considering the fact that many older adults with mild cognitive impairment progress to develop dementia, ${ }^{60}$ interventions that delay this progression are increasingly important. Improving self-efficacy for cognitive tasks in itself may reduce disability and dependency and improve quality of life.

Evaluation of increases in functional performance in relation to costs for residents, their families, and the health care system are also important to evaluate in future research. Cost analyses from this study that examine the cost for providing REAL in AL in relation to relative changes in the primary outcome scores are forthcoming, and pilot testing of REAL in small groups (a less-costly presentation format) will also be reported. ${ }^{61}$

\section{Limitations}

Some of the positive findings may actually represent practice effects that might be expected when measurements are repeated with the same assessment tools; however, the data did not support this conclusion. Repeated assessments in the no-treatment control group yielded no practice effect. It is likely that the 4- and 12-week intervals were spaced far enough apart that participants would be unlikely to remember specific items in the measures.

The findings, however positive, must be interpreted in light of certain limitations. The number of facilities was small, so the study had limited power and included facilities that 
self-selected to participate, and lower-quality facilities may not be reflected in this group, biasing the results. In addition, all facilities were in Kansas, and, considering differences in AL regulations by state, may not be generalizable to AL facilities across the country. Future studies should include the additional facilities needed to establish whether these findings are generalizable. Furthermore, it was impossible to complete data collection on residents who were not in the facility at follow-up, making a true intention-to-treat analysis not possible. In addition, participation rates in each facility varied, depending on how much support was offered by facility administrators and staff.

\section{Future research}

On the basis of the findings, additional longitudinal research, using a larger sample, is indicated. Moreover, the data suggest it might be beneficial to increase the number of sessions or add booster sessions. In addition, integrating a physical activity intervention may provide an additive or synergistic effect with cognitive training. ${ }^{62}$

It may be feasible to provide REAL training in groups, rather than in personal training sessions, as a cost-saving method. Research should also evaluate translation and implementation, using approaches such as "train-thetrainer", which would use paraprofessional staff at AL facilities.

Future research should also take into consideration changes in the level of acuity of AL residents that indicate residency by older adults with more cognitive and physical disabilities and a recent trend toward aging in place in AL. ${ }^{63,64}$ It would be key to evaluate whether REAL training would affect these AL industry trends.

\section{Conclusion}

REAL training led to gains in everyday problem-solving and functional performance for AL residents who varied in age and baseline cognitive status. If ongoing research can replicate the gains in everyday problem solving and self-care produced in this pilot study, support for cognitive training should be considered as a reimbursable service under Medicare, Medicaid, and private insurance. One option is to train certified nursing assistants to provide REAL or a similar cognitive program. A "cognitive" aide (similar to a rehabilitation or medication aide) in each AL facility could support a number of residents. It will be critical to demonstrate long-term outcomes for cognitive training in AL, (eg, extended AL stays and reduced NH placement rates) to justify reimbursement and build this new level of service.

\section{Acknowledgments}

The project described was supported by Award Number R21NR010880 from the National Institute Of Nursing Research. The content is solely the responsibility of the authors and does not necessarily represent the official views of the National Institute Of Nursing Research or the National Institutes of Health. DB received support from the National Institute of Deafness and Communication Disorders (grant P30DC05803).

\section{Disclosure}

The authors report no conflicts of interest in this work.

\section{References}

1. Golant SM. Do impaired older persons with health care needs occupy U.S. assisted living facilities? An analysis of six national studies. J Gerontol B Psychol Sci Soc Sci. 2004;59(2):S68-S79.

2. Mollica R, Johnson-Lemarche H. State residential care and assisted living policy: 2004. Washington, DC: US Department of Health and Human Services; 2005. Available from: http://aspe.hhs.gov/daltcp/ reports/2005/04alcom.htm. Accessed May 24, 2007.

3. National Center for Assisted Living. Resident profile. Available from: http://www.ahcancal.org/ncal/resources/Pages/ResidentProfile.aspx. Accessed May 24, 2007.

4. Maas ML, Buckwalter KC. Providing quality care in assisted living facilities: Recommendations for enhanced staffing and staff training. J Gerontol Nurs. 2006;32(11):14-22.

5. National Center for Assisted Living. Guiding Principles for Assisted Living. 2008. Available from: http://www.ahcancal.org/ncal/about/ Documents/GPAssistedLiving.pdf. Accessed May 16, 2014.

6. Kane RA, Wilson KB, Spector W. Developing a research agenda for assisted living. Gerontologist. 2007;47 Spec No 3:141-154.

7. Alzheimer News 3/18/2008 [webpage on the Internet]. Alzheimer's Association; 2008. Available from: http://www.alz.org/news_and_ events_13106.asp. Accessed May 19, 2014.

8. Hyde J, Perez R, Forester B. Dementia and assisted living. Gerontologist. 2007;47 Spec No 3:51-67.

9. Redfoot D, Feinberg L, Houser A. The Aging of the Baby Boom and the Growing Care Gap: A Look at Future Declines in the Availability of Family Caregivers. Washington, DC: AARP Public Policy Institute; 2013. Available from: http://www.aarp.org/content/dam/aarp/research/ public_policy_institute/ltc/2013/baby-boom-and-the-growing-caregap-insight-AARP-ppi-ltc.pdf. Accessed May 16, 2014.

10. Tabert MH, Albert SM, Borukhova-Milov L, et al. Functional deficits in patients with mild cognitive impairment: prediction of AD. Neurology. 2002;58(5):758-764.

11. Aud MA, Rantz MJ. Admissions to skilled nursing facilities from assisted living facilities. J Nurs Care Qual. 2005;20(1):16-25.

12. Dobbs-Kepper D, Chapin R, Oslund P, Rachlin R, Stover J. Resident and Facility Factors Related to Residents' Length of Stay In Assisted Living and Residential Health Care Facilities: A Longitudinal Analysis. Lawrence, KS: University of Kansas School of Social Welfare; 2001.

13. Phillips CD, Hawes C, Spry K, Rose M. Residents leaving assisted living: descriptive and analytic results from a national survey. Washington, DC: US Department of Health and Human Services; 2000. Available from: http://aspe.hhs.gov/daltcp/reports/2000/alresid.htm. Accessed June 30, 2008.

14. Williams KN, Warren CA. Communication in Assisted Living. J Aging Stud. 2009;23(1):24-36.

15. Williams KN, Warren CA. Assisted living and the aging trajectory. $J$ Women Aging. 2008;20(3-4):309-327. 
16. Hawes C, Phillips C, Rose M. High service or high privacy assisted living facilities, their residents and staff: results from a national survey. Washington, DC: US Department of Health and Human Services; 2000. Available from: http://aspe.hhs.gov/daltcp/reports/hshp.htm. Accessed May 15, 2007.

17. Zimmerman S, Sloane PD, Eckert JK, et al. How good is assisted living? Findings and implications from an outcomes study. J Gerontol B Psychol Sci Soc Sci. 2005;60(4):S195-S204.

18. Genworth Financial. Cost of Care. 2012. Available from: https://pro genworth.com/content/etc/medialib/genworth_v2/pdf/ltc_cost_of_care. Par.14625.File.dat/2010_Cost_of_Care_Survey_Full_Report.pdf. Accessed September 3, 2010.

19. Willis SL. Cognition and everday competence. In: Schaie KW, editor. Annual Review of Gerontology and Geriatrics. New York: Springer 1991.

20. Lawton MP, Brody EM. Assessment of older people: self-maintaining and instrumental activities of daily living. Gerontologist. 1969;9(3): 179-186.

21. Denney NW, Pearce KA. A developmental study of practical problem solving in adults. Psychol Aging. 1989;4(4):438-442.

22. Diehl M, Willis SL, Schaie KW. Everyday problem solving in older adults: observational assessment and cognitive correlates. Psychol Aging. 1995;10(3):478-491.

23. Schaie KW, Willis SL, Boron J. Everyday competence. In: Johnson M, editor. The Cambridge Handbook of Age and Ageing. London: Cambridge University Press; 2005.

24. Royall DR, Lauterbach EC, Kaufer D, Malloy P, Coburn KL, Black KJ; Committee on Research of the American Neuropsychiatric Association. The cognitive correlates of functional status: a review from the Committee on Research of the American Neuropsychiatric Association. J Neuropsychiatry Clin Neurosci. 2007;19(3):249-265.

25. Sitzer DI, Twamley EW, Jeste DV. Cognitive training in Alzheimer's disease: a meta-analysis of the literature. Acta Psychiatr Scand. 2006; 114(2):75-90.

26. Kang H, Smith M, Buckwalter KC, Ellingrod V, Schultz SK. Anxiety, depression, and cognitive impairment in dementia-specific and traditional assisted living. J Gerontol Nurs. 2010;36(1):18-30.

27. Baron RM, Kenny DA. The moderator-mediator variable distinction in social psychological research: conceptual, strategic, and statistical considerations. J Pers Soc Psychol. 1986;51(6):1173-1182.

28. Galvin JE, Roe CM, Coats MA, Morris JC. Patient's rating of cognitive ability: using the AD8, a brief informant interview, as a self-rating tool to detect dementia. Arch Neurol. 2007;64(5):725-730.

29. Folstein MF, Folstein SE, McHugh PR. "Mini-mental state". A practical method for grading the cognitive state of patients for the clinician. J Psychiatr Res. 1975;12(3):189-198.

30. Williams KN. Reasoning Exercises in Assisted Living (REAL): a feasibility study. Issues Ment Health Nurs. 2008;29(8):797-816.

31. Willis SL, Tennstedt SL, Marsiske M, et al; ACTIVE Study Group. Long-term effects of cognitive training on everyday functional outcomes in older adults. JAMA. 2006;296(23):2805-2814.

32. Charness N. Aging and problem-solving performance. In: Charness N, editor. Aging and Human Performance. Chichester, UK: John Wiley and Sons; 1985:225-259.

33. Smith CE, Leenerts MH, Gajewski BJ. A systematically tested intervention for managing reactive depression. Nurs Res. 2003;52(6): 401-409.

34. Tosi DJ, Rudy DR, Lewis J, Murphy MA. The psychobiological effects of cognitive experiential therapy hypnosis, cognitive restructuring, and attention placebo control in the treatment of essential hypertension. Psychother Theory Res Pract Train. 1992;29(2):274-284.

35. Shadish WR, Cook TD, Campbell DT. Experimental and QuasiExperimental Designs for Generalized Causal Inference. Boston: Houghton-Mifflin; 2002.

36. Burgio LD, Fisher SE, Phillips LL, Allen RS. Establishing treatment implementation in clinical research. Alz Care Quart. 2003;4(3):204-215.
37. Kolanowski A, Buettner L, Moeller J. Treatment fidelity plan for an activity intervention designed for persons with dementia. Am J Alzheimers Dis Other Demen. 2006;21(5):326-332.

38. Resnick B, Inguito P, Orwig D, et al. Treatment fidelity in behavior change research: a case example. Nurs Res. 2005;54(2):139-143.

39. Willis SL, Allen-Burge R, Dolan MM, Bertrand RM, Yesavage J, Taylor JL. Everyday problem solving among individuals with Alzheimer's disease. Gerontologist. 1998;38(5):569-577.

40. Centers for Disease Control and Prevention, National Center for Health Statistics. Home Health and Hospice Care Agencies: 2007 National Home and Hospice Care Survey. 2007. Available from: http://www.cdc. gov/nchs/data/nhhcs/2007hospicecaresurvey.pdf. Accessed May 16, 2014.

41. Bertrand RM, Willis SL. Everyday problem solving in Alzheimer's patients: a comparison of subjective and objective assessments. Aging Mental Health. 1999;3(4):281-293.

42. Bertrand RM, Willis SL, Sayer A. An evaluation of change over time in everyday cognitive competence among Alzheimer's patients. Aging Neuropsychol Cogn. 2001;8(3):192-212.

43. Loewenstein DA, Argüelles S, Bravo M, et al. Caregivers' judgments of the functional abilities of the Alzheimer's disease patient: a comparison of proxy reports and objective measures. J Gerontol B Psychol Sci Soc Sci. 2001;56(2):P78-P84.

44. Zanetti O, Frisoni GB, Rozzini L, Bianchetti A, Trabucchi M. Validity of direct assessment of functional status as a tool for measuring Alzheimer's disease severity. Age Ageing. 1998;27(5):615-622.

45. Loewenstein DA, Amigo E, Duara R, et al. A new scale for the assessment of functional status in Alzheimer's disease and related disorders. J Gerontol. 1989;44(4):P114-P121.

46. Loewenstein DA, Rubert MP. Staging functional impairment in dementia using performance-based measures: a preliminary analysis. J Mental Health Aging. 1995;1:47-56.

47. Teng EL, Chui HC. The Modified Mini-Mental State (3MS) examination. J Clin Psychiatry. 1987;48(8):314-318.

48. Jones AL, Harris-Kojetin L, Valverde R. Characteristics and Use of Home Health Care by Men and Women Aged 65 and Over. 2012;52. Available from: http://www.cdc.gov/nchs/data/nhsr/nhsr052.pdf. Accessed May 16, 2014.

49. Spitzer RL, Kroenke K, Williams JB. Validation and utility of a selfreport version of PRIME-MD: the PHQ primary care study. Primary Care Evaluation of Mental Disorders. Patient Health Questionnaire. JAMA. 1999;282(18):1737-1744.

50. Nezlek JB, Schroder-Abe M, Schutz A. Multilevel analyses in psychological research. Advantages and potential of multilevel random coefficient modeling. Psychologische Rundschau. 2006;57(4):213-223.

51. Misangyi VF, LePine JA, Algina J, Goeddeke F. The adequacy of repeated-measures regression for multilevel research - Comparisons with repeated-measures ANOVA, multivariate repeated-measures ANOVA, and multilevel modeling across various multilevel research designs. Org Res Methods. 2006;9(1):5-28.

52. Gueorguieva R, Krystal JH. Move over ANOVA: progress in analyzing repeated-measures data and its reflection in papers published in the Archives of General Psychiatry. Arch Gen Psychiatry. 2004;61(3): 310-317.

53. Cnaan A, Laird NM, Slasor P. Using the general linear mixed model to analyse unbalanced repeated measures and longitudinal data. Stat Med. 1997;16(20):2349-2380.

54. Murray DM, Hannan PJ, Wolfinger RD, Baker WL, Dwyer JH. Analysis of data from group-randomized trials with repeat observations on the same groups. Stat Med. 1998;17(14):1581-1600.

55. Marsh LC, Cormier DR. Spline Regression Models. Quantitative Applications in the Social Sciences. Series 07-137. Thousand Oaks, CA: Sage Publications; 2002.

56. Gould WW. Linear splines and piecewise linear functions. Stata Tech Bull. 1993. Available from: http://www.stata.com/products/stb/journals/ stb15.pdf. Accessed November 28. 
57. Panis C. The piecewise linear spline transformation. Stata Tech Bull. 1994. Available from: http://www.stata.com/products/stb/journals/ stb18.pdf. Accessed November 28.

58. Salthouse TA. Mental exercise and mental aging: Evaluating the validity of the "use it or lose it" hypothesis. Perspectives Psychol Sci. 2006;1(1):68-87.

59. Thompson G, Foth D. Cognitive-training programs for older adults: what are they and can they enhance mental fitness? Educ Gerontol. 2005;31(8):603-626.

60. Petersen RC. Mild cognitive impairment as a diagnostic entity. J Intern Med. 2004;256:183-194.

61. Lustig TA, Olsson S. Financing long-term services and supports for individuals with disabilities and older adults: Workshop summary. 2013. Available from: http://www.nap.edu/catalog.php?record_id=18538. Accessed May 16, 2014.
62. Barnes DE, Santos-Modesitt W, Poelke G, et al. The Mental Activity and eXercise (MAX) trial: a randomized controlled trial to enhance cognitive function in older adults. JAMA Intern Med. 2013;173(9): 797-804.

63. Harris-Kojetin L, Sengupta M, Park-Lee E, Valverde R. Long-Term Care Services in the United States: 2013 Overview. Hyattsville, MD: National Center for Health Statistics; 2013. Available from: http://www. cdc.gov/nchs/data/nsltcp/long_term_care_services_2013.pdf. Accessed May 16, 2014.

64. Allen J; American Assisted Living Nurses Association Board Member. Higher acuity in assisted living is here to stay. Geriatr Nurs. 2011;32(1):69-71.
Clinical Interventions in Aging

\section{Publish your work in this journal}

Clinical Interventions in Aging is an international, peer-reviewed journal focusing on evidence-based reports on the value or lack thereof of treatments intended to prevent or delay the onset of maladaptive correlates of aging in human beings. This journal is indexed on PubMed Central, MedLine,

\section{Dovepress}

CAS, Scopus and the Elsevier Bibliographic databases. The manuscript management system is completely online and includes a very quick and fair peer-review system, which is all easy to use. Visit http://www.dovepress. com/testimonials.php to read real quotes from published authors. 